\title{
Cognitive Psychology Models and Approaches to Develop Language Skills
}

\author{
Imad Tahini, Tomokazu Nakayama, Vitaly Dibrova, and Alex Dadykin
}

\begin{abstract}
There is a need to raise the quality of communication between people that are speaking different languages to a higher level in the modern world. This requires finding new ways to improve language learning and teaching. The basis of the conducted researches is made by cognitive psychology models. The last developments are directed to the association of two new methods, simultaneous development of language skills and grammatical competence of one activity. The methods are the Visual-Auditory Shadowing (VAS) method and the Structural-Visual Method (SVM). The Structural-Visual Method is a new inductive language learning methodology, based on mapping of the structure of linguistic knowledge in a graphic form using color to encode the most common patterns. The Visual-Auditory Shadowing method is also an inductive language learning methodology, which facilitates learning of phonological knowledge (pronunciation) and ideographical knowledge (spelling). Each of these methods can be improved through the use of computer systems and the combined use of both will lead to a synergistic effect in the learning process. The study provides a conceptual solution which requires experimental verification and further joint research.
\end{abstract}

Index Terms-Learning management system, model of the psyche, structural-visual method, visual Model, visual-auditory shadowing method.

\section{INTRODUCTION}

Studies in the field of cognitive psychology, linguistics and pedagogy have not yet led to the creation of generally accepted models of language learning processes and the acquisition of language skills.

Even greater disagreements are observed in the description of the whole complex of processes that occur with the participation of the language - thinking, consciousness, memory, and the totality of mental processes as a whole. These processes are very complex, multilevel and dynamic.

To harmonize the terminology and structure of the description of mental processes in interdisciplinary projects, the paper [1] proposes a minimal 4-element model of the structure of mental processes, and a 3-level model for the interaction of the psyche with reality. Due to their simplicity, these models can serve as a starting point for reconciling more detailed models of each of the elements and correlating

Manuscript received December 29, 2017; revised March 12, 2018.

I. Tahini is with the Belarusian National Technical University, Beirut, Lebanon (e-mail: imad.tahini@live.com)

T. Nakayama is with the Jissen Women's University, Tokyo, Japan (e-mail: nakayama-tomokazu@jissen.ac.jp)

V. Dibrova is with the Languages Studio, Kiev, Ukraine (e-mail: tivita@mail.ru)

A. Dadykin is with the Belarusian National Technical University, Minsk, Belarus (e-mail: alex_05_07@rambler.ru) models in different branches of knowledge with each other.

Applying these models as a functional structure of the highest level of abstraction, one can find a place for all the proposed models of cognitive science, by decomposing the elements of this model to the required detail.

Let us compare, for example, Baddeley's model of working memory [2], which plays an important role in the analysis of the processes of primary understanding, memorization, and further training and skills formation (Fig. 1).
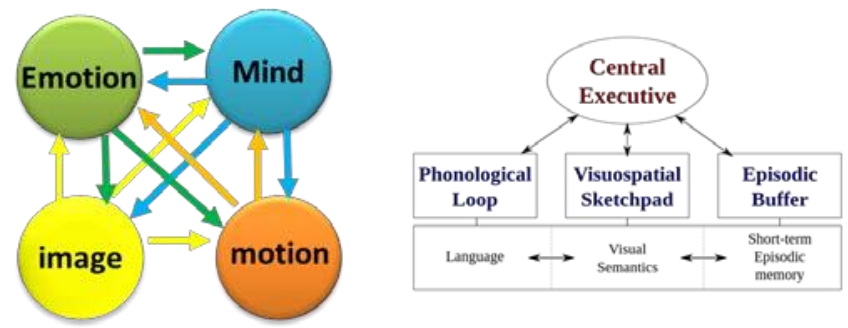

Fig. 1. A generalized model of the psyche and a model of working memory of Baddeley.

To relate these two models, we will make the decomposition of the verbal mind into components, of which language skills are composed - images of the sounds of the language, movements for pronouncing these sounds, images of letters (words) and images of movements for writing (or printing) these letters (stored in long-term memory words) (Fig. 2)

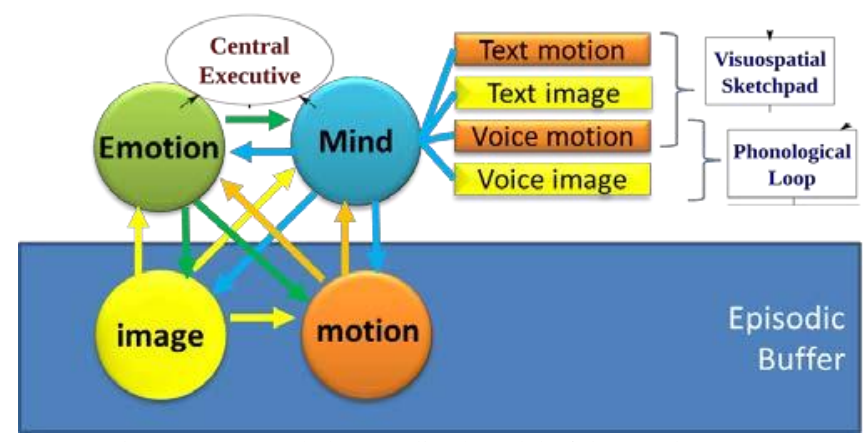

Fig. 2. An expanded generalized model of the psyche.

Thus, we see the following correspondence of the elements of these models. The phonological loop corresponds to the connection between sound and the image of movement, the visual notepad - the connection of the image of the sign (word) with the images of movements, the episodic buffer corresponds to the primary sensory and motor information in the corresponding zones of the brain, and the processes of motivation, attention and interest are determined by the connections cerebral cortex with the limbic system.

As was shown in the works of Ivanitsky [3], Edelman [4] 
and other researchers, information processes in the brain are to re-projection memory contents into the zone of reception of the primary signal. Those are the very perception, awareness, understanding arises at the time the neural self-sustaining loop emerges (Fig. 3).

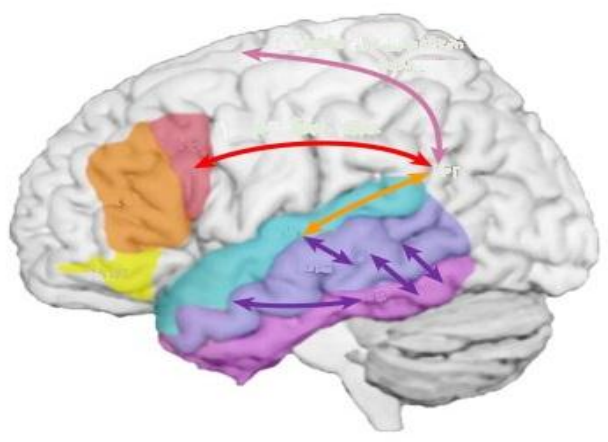

Fig. 3. Sensory-motor loop.

Dominant excitation of such a loop is the basis of the processes of attention, consciousness, awareness. Such a model removes the problem of the "homunculus", which "receives", "extracts", "presents" information from the organs of perception or memory. In fact, there is no "extraction from memory", signals coming from the perception organs find a response in the areas of the brain that have accumulated information about similar processes, and the return of this information to the primary projection zone forms a "model of the current situation", or "episodic buffer" in terms of the Baddeley model.

This understanding of the processes of perception and memory allows a different look at learning and mastering a foreign language, and find new, faster and more effective ways for this.

\section{NEW METHOdS OF IMPROVING LISTENING SKILLS}

The main goal of training, as defined by CEFR [5], is to achieve competence in the language. Competences of the pupil develop in the process of using the language and allow you to perform communicative tasks and actions in situations of communication in different conditions and contexts.

One of the most challenging tasks is the Improving listening comprehension in an EFL, especially for languages with great the phonological differences between the learners' first language and English.

To study the measurement and management of the Improving listening comprehension process, it is very important to have a scientific model of this process in order to isolate and investigate the individual impact of each of the significant process parameters and to exclude the influence of non-significant.

At the moment, there are more than 10 theories of speech perception (Exemplar theory, Motor theory, Direct realist theory, Cohort model etc.), several well-designed models from Russian scientists (Leontiev [6], Chistovich [7], Zimnjaja [8] and etc.).

For practical purposes, you can use the simple model shown in Fig. 3.

What conclusions can be drawn from this model, taking into account the theory of repeated input?

Simply listening to language samples outside of communication with other cognitive and linguistic processes will be extremely inefficient or generally useless.

To form the skills of understanding what is heard, it is necessary to develop a phonological loop, i.e. communication of auditory images with speech movements. One of the best approaches for this, it is the shadowing method. Shadowing is "an act or a task of listening in which the learners track what he/she heard in speech and repeats it as accurately as possible while listening attentively to the in-coming information (p.34) [9]" according to Tamai (2005), the shadowing method is effective in improving phonological loop process, and it leads to improvement in listening skills.

Since there are two variants of linguistic activity (and two kinds of sign RAM), a significant acceleration can be achieved using both of these channels simultaneously, as proposed in the variant of improving this approach - Visual Auditory (VA) shadowing.VA shadowing method is a combination of auditory shadowing and visual shadowing. Auditory shadowing requires so-called online processing of auditory input, which requires listening and repetition at the same time. Visual shadowing requires learners to read text aloud employing online processing [10] according to Nakayama (2017), VA shadowing method better improves learners' listening skills, compared to shadowing method alone.

If the pupil lacks sound images and motor skills of a new language, he will use for these purposes the corresponding resources of his native language. In some cases, such a strategy will be sufficient to solve limited communication problems, so this option of using the language (known as "international English") has the right to exist and develop.

As studies by Kuhl [11] have shown, the formation of acoustic images of the sounds of the native language occurs quickly, in a very short sensitive period from 7 to 9-10 months development of the child. In the future the mechanism of primary education is replaced by the process of retraining, overtraining, which has quite different patterns. Taking into account these patterns can significantly improve the understanding and pronunciation skills in adults, especially for languages with other phonetics.

Taking into account point 5 , the number of repetitions of listening and pronouncing the same material used in most methods and approaches is completely inadequate for such retraining. It requires 5-30 times more repetitions than the development of the primary skill (Pavlov [12], Skinner [13], and Bandura [14]).

Multiple listening to the same material has a very negative impact on motivation, and requires a lot of strong-willed efforts. In addition, this is inefficient from the point of view of the formation of broad auditory images, which allow one to understand different voices and accents. Therefore, the concept of "Repetition without repetition", where each presentation of the same material is produced by different voices, with different speed, tone, intonation and accent, is of interest. In this case, an important role is played by the use of technical means to implement such a task.

1) For more rapid formation of sound filters and pronunciation patterns, it is proposed to use the 
technologies Text-to-Speech, voice recognition, audio file decoding and other achievements of computer technology.

2) The need for multiple repetitions can be used to simultaneously develop both speech skills and linguistic competencies. For this purpose, a combination of the VA shadowing technique, enhanced by the use of computer technologies, and the technique of Structural-Visual modeling.

The SVM methodology replaces the verbal description of the language with colored diagrams, which allows us to use in parallel the other channels of the psyche - visual-spatial and color (included in the Episodic Buffer in Fig. 3).

\section{NEW METHODS OF IMPROVEMENT OF GRAMMATICAL SKILLS (COMPETENCIES)}

When researching the ways to accelerate the achievement of competence in certain aspects of the language, it is important to take into account the theories of cognitive psychology, which theoretically substantiate the laws of this process.

Galperin in his theory of the step-by-step formation of mental actions [15] pointed to the need for an indicative stage in mastering the skill of mental action, emphasizing the importance of auxiliary tools that facilitate this orientation the so-called schemes of the Basis of Activity.

A similar approach in Western pedagogy is widely known as Instructional scaffolding. This theory was developed around the same time by Bruner [16].

Both these theories are based on Vygotsky's idea of a zone of proximal development [17]. Additional support tools and instructions can be provided through the sensory, motor and verbal channels.

But the application of grammatical rules as such an indicative basis, support (scaffolding) for the development of skills in linguistic activity is difficult because the understanding and application of these rules is the same language activity, which at this time should be trained on the basis of the communicative situation. The same resources of the psyche and memory cannot be used simultaneously to carry out the activity and management of the same activity.

A similar phenomenon was discovered in the experiments of Zhinkin [21] and Chistovich [7] in the middle of the last century, and the low effectiveness of grammatical rules in the mastering of oral speech was repeatedly declared by many linguists and psychologists (Krashen [18], Pinker [19] and etc.).

To resolve this contradiction, it is proposed to apply the SVM.

The SVM in linguistics is a mapping of the structure of linguistic knowledge in a graphic form using the color of visual objects to encode the most common patterns. The models thus obtained replace the textual explanations (rules) in the formation of the corresponding skills.

The higher efficiency of this method compared to other Visual Aids follows from the features of the functioning of the human visual system, shown in recent studies by Kozlovsky [20].
In them, the conceptual model of Baddeley's work memory was confirmed at the physiological level, in particular the part where the presence of visual working memory is declared, which functions independently of verbal working memory ("visual-spatial matrix").

Experiments due to Kozlovsky demonstrated not only the presence of such memory, but also determined what structure of the brain it provides. And it was found that the working memory of the spatial characteristics of the stimulus and working memory of its color and shape is provided by different brain systems.

The presence of two independent visual information processing systems makes it possible to simultaneously encode one type of regularities by using the structure and form of visual objects, and the other by using color, as is done in the proposed SVM. In this case, it is possible to easily show and explain regularities that are difficult to explain with the help of words because of the limitation on the amount of verbal RAM of the student (according to the model of Baddeley).

For the application of the SVM in linguistics, it is proposed to encode information on the method of constructing an English sentence using spatial placement and the form of visual objects, and about temporal forms and related senses-using spatial placement and color.

The color values are learned in advance by the pupil with the help of the native language on illustrative examples. At the same time, the meaning is transferred from the language to the intermediate visual sign system, which allows the student to understand the meaning of perceived or produced constructions in the studied language without reliance on the native language.

Models contain the necessary set of temporary forms and constructs of the types of supply required for this level of language proficiency or according to the curriculum of this training course. For example, the simplest model for constructing a simple narrative sentence in 4 time forms for an action verb is shown in Fig. 4.

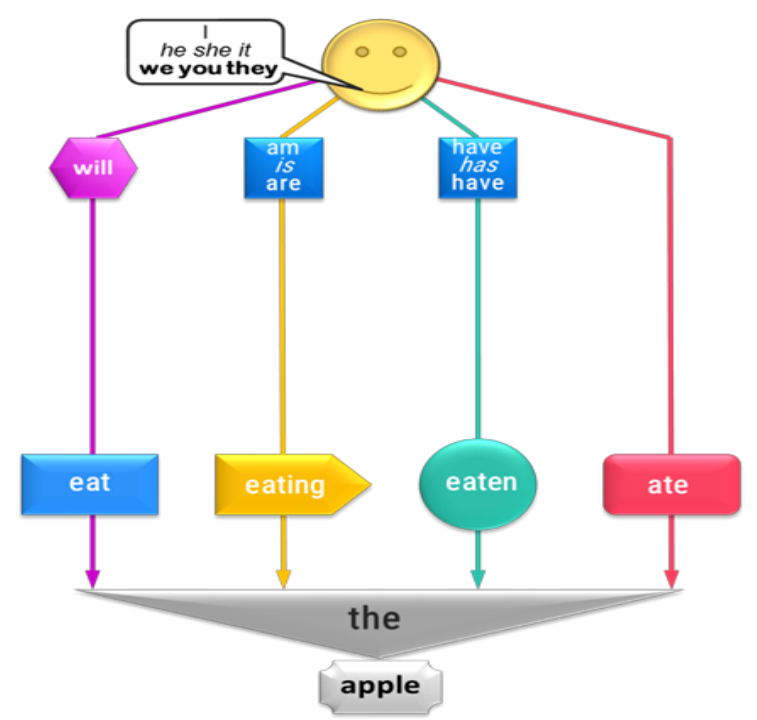

Fig. 4. Model of a simple narrative sentence for a verb of action.

Fig. 5 shows a more detailed model that allows you to build on it proposals of different forms (narrative, interrogative, 
negative) and for more times.

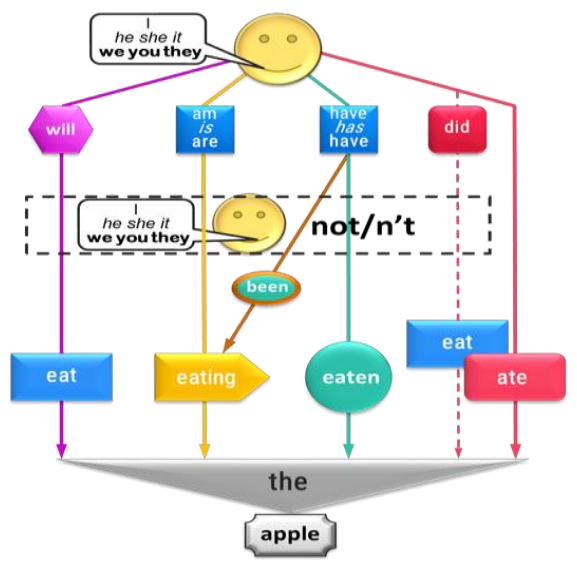

Fig. 5. Model for different forms of sentences with action verbs.

In Fig. 6 a similar model is given for the verbs of state (Stative Verbs).

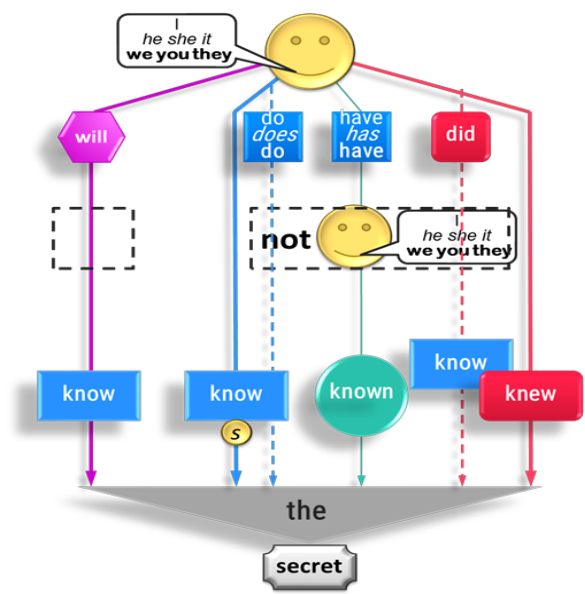

Fig. 6. Model for different forms of sentences with state verbs.

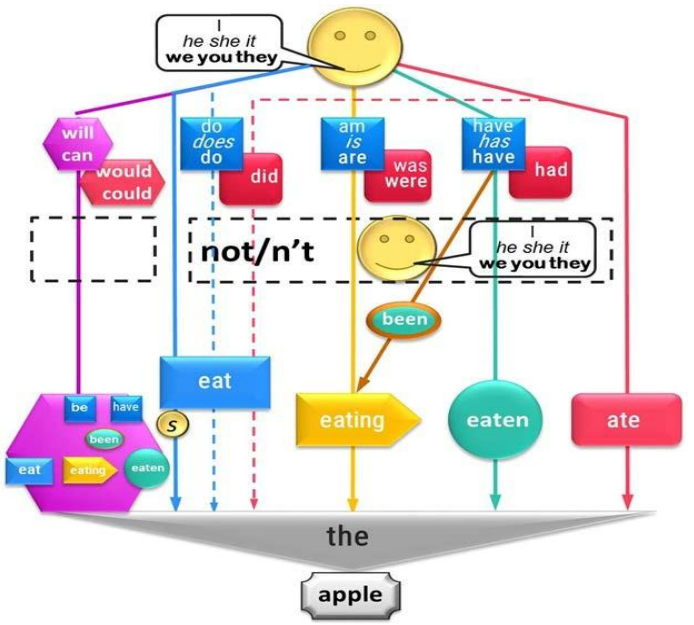

Fig. 7. Full model of all active lien times.

In Fig. 7 shows the full model of all types of active forms of active voice. It can be applied at higher levels of grammatical competence to systematize knowledge and understand the complete structure of a system of times.

Also, models have been created to study individual grammatical topics - passive voice, modal verbs, impersonal verbs, conditional sentences, and many other difficult to understand topics.

This approach allows us to start the mechanism from the first sessions, directly linking the sentences of the language being studied to the meaning behind them, and to exclude negative interference from the structure of the native language, which in many cases may differ significantly from the target language.

\section{APPLICATION FOR IT DEVELOPMENT OF LANGUAGE SKILLS}

Mastering a foreign language is a complex and time-consuming task for both students and organizers of the learning process, as the language, unlike other subjects, is a complex multilevel system that includes, in addition to knowledge of the subject, a large number of practical skills and skills.

Therefore, modern computer and IT technologies can greatly help in improving the effectiveness of learning processes for students and reducing the burden on teachers, but for this it is necessary to ensure mutual understanding among specialists in different fields of activity, harmonizing terminology and models of the subject field.

Since it is necessary to ensure mutual understanding and joint work of practical teachers, methodologists, psychologists, mathematicians, programmers, IT engineers and other specialists very far from each other in the various fields of knowledge to create computer programs and systems for managing the learning process and skills acquisition.

The problem is complicated by the fact that in different countries in each of these branches terminology is used in different languages and is based on scientific models and paradigms of different structures.

To eliminate these problems, it is proposed to use the terminology and taxonomic scheme, developed by a large team of scientists for many decades in the process of work on a document of the Council of Europe CEFR, as a framework for joint projects. And to simplify the understanding of a particular problem to be solved, it is widely used the SVM, as proposed in [1].

The document of the Council of Europe is carefully worked out, comprehensive and consistent. It takes into account all stakeholders, all the main elements and parameters of the process. But due to the complexity of the language system, the process of its study is also very complex and contains dozens of elements and hundreds of mutually affecting parameters.

To create a technical system that manages such a process and takes into account all these elements and parameters, it is not possible at the present level of technological development. But to manage the process of acquiring individual elements of the language or mastering individual skills, the possibilities have existed for a long time.

In recent years, there has been a qualitative leap in voice, speech technologies, cloud computing, big data, machine learning and neural networks. There were prerequisites for using these technologies both to facilitate the work of teachers, and to optimize and accelerate the learning processes, and especially the acquisition of skills.

The basis for the harmonization of terminology, methods, procedures and structures in the process of creating a new generation of Learning Management System (LMS) is a 
visual meta-language tools and systems analysis, as a link between theory and practice, IT and humanities field, science and social processes (Fig. 8).

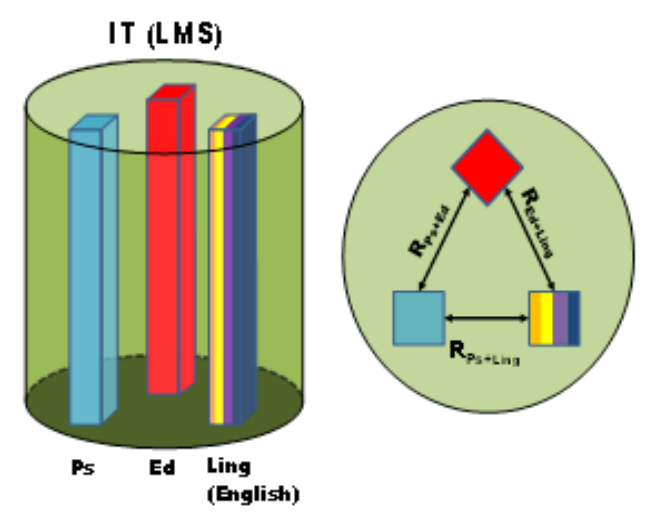

Fig. 8. Structural diagram of LMS (upper level of abstraction), where: Ed - Education; Ps - Psychologic; Ling - Linguistic.

The authors recommend a conceptual solution in the form of the creation of a training system [1], which allows to control the process of formation of language and work skills of adults. The generalized structure of the new generation LMS is shown in Fig. 9.

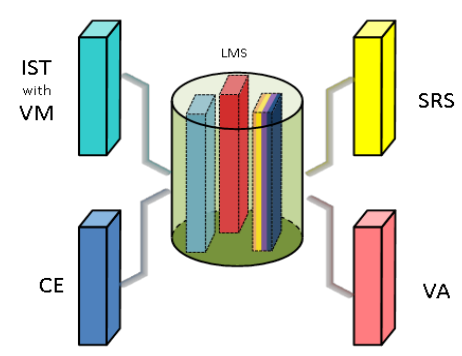

Fig. 9. LMS Generalized structure, where: IST with VM - Interactive speech trainers with visual models, SRS - Speech recognition system, CE continuous evaluation, VA - virtual assistant.

This system combines:

1) Methodical principles substantiated in the works of Bandura [14] and Galperin [15].

2) Unification of visual models and interactive visual-auditory tracking, creating a synergistic effect, both in the initial phase of mastering a foreign language, and at the stage of the "barrier of overcoming".

3) Use the achievements of the IT-sphere as a tool that ensures the implementation of learning objectives with continuous monitoring of the current state and obtaining a guaranteed learning outcome in a finite number of steps.

4) Further development of the main components of the LMS is carried out in the following interrelated areas.

5) It is proposed to use SVM in combination with the modified VAS method. Using a new type of LMS provides:

- Step-by-step deceleration and acceleration of the speed of speech in a programmatic way to facilitate the formation of correct pronunciation.

- Application of reference sound templates and speech simulators.

- Repetition without repetition - use for each approach of a material changed by some parameter (voice, tempo, height, lexical or grammatical transformations).

The joint use of these elements will allow you to repeatedly switch the visual, auditory and emotional memory during the lesson, thus allowing better, and most importantly, less effort to memorize the material being studied. Such a method can be called mini-max, when the task of obtaining the maximum possible result with the minimum expenditure of forces is realized. In the process of learning, the unnecessary work that does not have a subsequent result is completely excluded. Only that which gives the result is performed, and this effect is felt by the student immediately, which gives an unconditional stimulus to further studies and increases the motivation for learning.

Setting up a Continuous Evaluation subsystem and managing the learning process in real time will allow us to form the logarithmic dependence of the learning curve and compensate the prerequisites for the degradation of the learning curve towards the loss of the expected competence.

The use of modern IT in combination with the use of effective models for the acquisition of skills allows to reduce or even completely eliminate the impact of various psychological obstacles and thereby accelerate learning and improve its success by transferring a synergistic effect to all stages of the formation of language skills, especially "barriers to overcome" (Fig. 10).

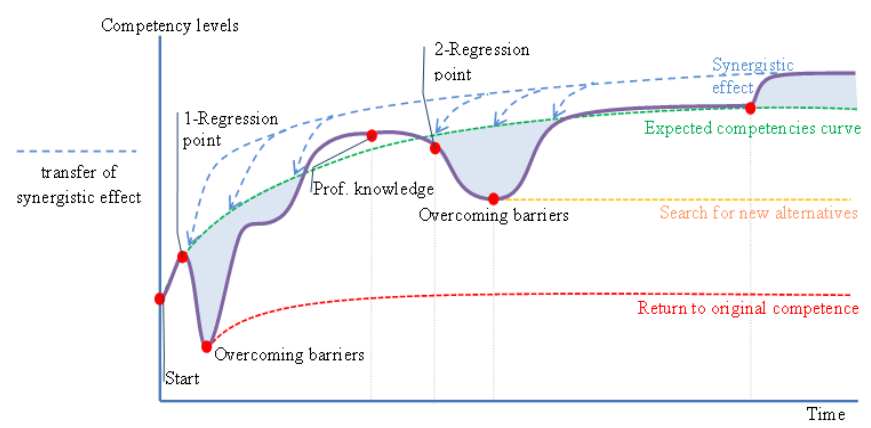

Fig. 10. The learning curve with the transfer of synergistic effect.

The training thus organized will provide a process of controlled formation of speech skills to a threshold level, allowing to move from studying the language to its improvement in the process of use. Measurement and investigation of numerical parameters of this process will allow obtaining the necessary data for building an automated control system for language acquisition, which, in fact, opens up a new direction of search in the construction of LMS.

\section{CONCLUSION}

In the work a study was carried out to identify some more particular problems in the general language acquisition process, two new very promising methods were analyzed and possible ways of their further research, improvement, integration and automation.

Particularly strongly the effect of the application of visual models and the SVM can manifest itself in the study of English by pupils with a native language, in which non-alphabetic writing, like Chinese or Japanese, is used. It 
seems that the carriers of these languages, by virtue of such a device of their writing, significantly develop their visual memory (visual-spatial matrix according to Baddeley) and visual-spatial thinking. But this is only an assumption that requires experimental verification and joint research. To do this, it is proposed to conduct a joint study involving students with the Latin alphabet of their native language (Spain, Hispanic students in the US), not the Latin alphabet (Ukraine, Belarus, Lebanon) and hieroglyphic writing (Japan, China). Comparing the obtained data on the speed of acquiring individual grammatical skills using Visual Models for these three groups of students can yield very valuable results and shed light on important aspects of the formation of linguistic and linguistic competencies.

\section{REFERENCES}

[1] A. K. Dadykin, V. A. Dibrova, and I. H. Tahini, "The visual approach in educational projects," International Journal of Social Science and Humanity, vol. 7, no. 6, pp. 373-377, June 2017.

[2] A. D. Baddeley, The Episodic Buffer: A New Component of Working Memory? Trends in Cognitive Sciences, no. 4, pp. 417-423, 2000.

[3] A. M. Ivanitsky, "Informational synthesis in crucial cortical area as the brain base of the subjective experience," Journal of Higher Nervous Activity them. I.P. Pavlov, vol. 47, no. 2, pp. 223-225, 1997.

[4] G. Edelman and G. Tononi, Consciousness. How Matter Becomes Imagination, L.: Penguin Books, 2000.

[5] Common European Framework of Reference for Languages: Learning, Teaching, Assessment, Council of Europe, 2011.

[6] A. A. Leontiev, Language and Speech Activity in Universal and Pedagogical Psychology, Moscow, MPSI, 2003, 536 p.

[7] L. A. Chistovich, Physiology of Speech, Perception of Human Speech, L.: Nauka, 1976, 388 p.

[8] I. A. Zimnjaja, The Sense Perception of a Speech Message, M.: Nauka, 1976, pp. 5-33.7.

[9] K. Tamai, Research on the Effect of Shadowing As a Listening Instruction Method, Tokyo: Kazama Shobo, 2005.

[10] T. Nakayama, Efficacy of Visual-Auditory Shadowing Method in SLA Based on Language Processing Models in Cognitive Psychology, Tokyo: Kaitakusha, 2017, 110 p.

[11] P. K. Kuhl, "Cognitive control skills and speech perception after short term second language experience during infancy," The Journal of the Acoustical Society of America, Jun 2008.

[12] I. P. Pavlov, Twenty-Year Experience of an Objective Study of the Higher Activity (Behavior) of Animals, Moscow: Nauka, 1973, $661 \mathrm{p}$.

[13] B. F. Skinner, The Technology of Teaching, New York, 1968.

[14] A. Bandura and R. H. Walters, Social Learning and Personality Development, New York: Holt, Rinehart, \& Winston, 1963.

[15] P. Y. Galperin, Psychology of Thinking and Teaching about the Gradual Formation of Mental Actions, Research in the thinking of Soviet psychology, Moscow, 1966

[16] A. Ninio and J. Bruner, "The achievement and antecedents of labeling," Journal of Child Language, no. 5, pp. 1-15, 1978.

[17] L. Vygotsky, Thought and Language, Cambridge, MA: MIT Press, 1934/1986.

[18] S. D. Krashen, Principles and Practice in Second Language Acquisition, University of Southern California, $202 \mathrm{p}$.

[19] S. Pinker, The Language Instinct: How the Mind Creates Language, William Morrow and Company, 1994, $483 \mathrm{p}$.

[20] S. A. Kozlovsky. Psychophysiological mechanisms of preservation of visual images in working memory. [Online]. Available: http://www.dissercat.com/content/psikhofiziologicheskie-mekhanizm y-so

khraneniya-zritelnykh-obrazov-v-rabochei-pamyati\#ixzz4zGVJphTy.

[21] N. Zhinkin, "About code transitions in internal speech," Questions of linguistics, no. 6, pp. 26-38, 1964.

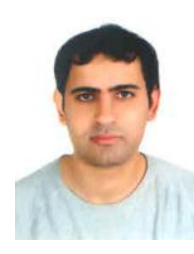

Imad H. Tahini was born in Aita el chaab, Lebanon, in 1981. From 1999 to 2006, he got a teaching diploma in applied mathematics option: computer science from Lebanese University faculty of science. From 2011 to 2012 he got a master degree in computer science and communication from Arts, Sciences And Technology University in Lebanon. Since 2016 he is a Phd student at Belarusian National Technical University.

From 2006 to 2018 he worked as a programmer, software development team leader and data analyst in HEC, HHC and Servo-pro Companies. he interested in developing business software and design for database methodology and he has a business knowledge in accounting and finance. $\mathrm{He}$ is the Member of IEEE. His current research is in the field of learning management system.

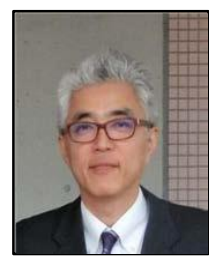

Tomokazu Nakayama A. was born and raised in Tokyo, Japan. Nakayama earned a bachelor degree in English literature and linguistics from Obirin University in 1991, and MA in TESOL at Teachers' College Columbia University in 2001 and Ph.D. at Hiroshima University in 2013. He is specialized in learning science. His current research interests are English as an international language (EIL) and development of new learning methods to promote proficiency of EIL learners. He developed VA shadowing method to improve Japanese EIL learners' listening skills and the book on its mechanism will be released this year. Now he and his colleagues are developing the new method called Instant Translation method to promote proficiency of Japanese EIL learners. $\mathrm{He}$ is currently a professor at Jissen Women's University in Tokyo and teaches English and English teacher training courses.

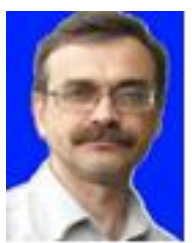

Vitaly A. Dibrova was born in Poltava reg., Ukraine, May 26, 1963

In 1986-1988 he served as an officer in Soviet Air Force. Since 1989 he worked as an engineer in the Science and Production Association "Karat". After collapse of the Soviet Union he worked in different fields of business.

In 2007 he launched the internet startup "Filolingvia" where he has been a CEO. For developing this project he was involved in independent scientific research in different field of science. He is the author of four scientific publications, and has two inventor's certificate.

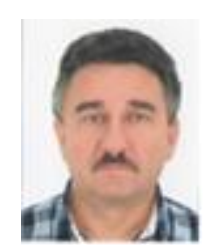

Alex K. Dadykin was born in Minsk. He received his master degree, $\mathrm{PhD}$ in automated control systems from Minsk High Engineering Military School of anti-aircraft missile defense graduated with honors, in 1980 and 1989 respectively. In 1991, he was awarded the degree of candidate of technical sciences (Soviet Union), and in 1999 was awarded the academic title of associate professor on "Informatics, control and computer engineering" (Republic of Belarus).

From 2001 to 2009, he served as scientific secretary of the Council for the defence of dissertations at the Military Academy of the Republic of Belarus. Currently, he is an associate professor of the Department of Information Systems and Technology of the International Institute of Distance Education and a Chairman of the scientific meeting at the Belarusian National Technical University. He is the developer of four of the State educational standards for higher education for practice-oriented master's degree.

Dr. Dadykin in 2009 was elected professor of the academy of military sciences of the Russian Federation. He is the author of more than seventy educational-methodical and scientific works, has five inventor's certificate. $\mathrm{He}$ is the Senior Member of IEDRC and the Member of IEEE. His main areas of research are in the field of system analysis, the development of learning management systems and mobile robotic systems. 